William M. Mendenhall, M.D., James T. Parsons, M.D., Scott P. Stringer, M.D., Nicholas J. Cassisi, D.D.S., M.D., George T. Singleton, M.D., and Rodney R. Million, M.D.

\title{
Radiotherapy in the Management of Temporal Bone Chemodectoma
}

\begin{abstract}
Forty patients with 42 temporal bone chemodectomas were treated with radiotherapy alone ( 37 tumors) or subtotal resection and irradiation ( 5 tumors) at the University of Florida between 1968 and 1992. Thirty-three lesions were previously untreated, whereas 9 had undergone prior treatment (surgery, 6 lesions; radiotherapy, 1 lesion; or both, 2 lesions) and were treated for locally recurrent disease. All 3 patients who received prior radiotherapy had been treated at other institutions. Patients had minimum follow-up times as follows: 2 years, 40 patients $(100 \%) ; 5$ years, 31 patients $(78 \%) ; 10$ years, 21 patients $(53 \%) ; 15$ years, 16 patients (40\%); 20 years, 9 patients $(23 \%)$; and 25 years, 2 patients $(5 \%)$. The local control rate at 20 years, calculated by the Kaplan-Meier product-limit method for the overall group of 42 lesions, was $89 \%$. The likelihood of cause-specific survival at 20 years was $94 \%$. The incidence of treatment-related complications was acceptable. We conclude that irradiation offers a high probability of tumor control with relatively minimal risks for patients with chemodectomas of the temporal bone. (Skull Base Surgery, 5(2):83-91, 1995)
\end{abstract}

Limited chemodectomas of the temporal bone may be managed with complete resection with a high probability of local control and a low risk of complications. ${ }^{1,2}$ In contrast, resection of extensive lesions may result in a higher likelihood of incomplete excision and substantial morbidity. ${ }^{3-10}$ This has prompted some to treat extensive lesions, as well as early lesions in patients judged to be medically infirm, with primary radiotherapy. $3,5,8,11-18$

Proponents of surgery argue that because chemodectomas usually remain stable or only partially regress after radiotherapy, the treatment is only palliative compared to an apparent complete resection. ${ }^{19}$ Additionally, they point out that the follow-up in many irradiation series is inadequate to define the risk of late recurrence. ${ }^{19}$

The purpose of this article is to review our experience with radiotherapy in the management of temporal bone chemodectomas and to discuss the relative advantages and disadvantages of radiotherapy compared with surgery.

\section{METHODS}

Forty patients with chemodectomas of the temporal bone were treated with radiotherapy at the University of Florida, between January 1968 and June 1992. Because two patients had synchronous bilateral temporal bone chemodectomas, 42 tumors were evaluable for local control. Patients had minimum follow-up times as follows: 2 years, 40 patients (100\%); 3 years, 34 patients $(85 \%)$; 5 years, 31 patients (78\%); 10 years, 21 patients $(53 \%) ; 15$ years, 16 patients (40\%); 20 years, 9 patients (23\%); and 25 years, 2 patients $(5 \%)$. Follow-up was calculated from the date of initiation of irradiation at the University of Florida for those patients treated with radiotherapy alone, and from the date of surgery for those undergoing subtotal resection and irradiation. Two patients who had no evidence of disease progression were lost to follow-up at 8 
years and 15 years, respectively. They were coded as dead of intercurrent disease at the date of last follow-up.

There were 28 women and 12 men; 25 patients were white, 14 were black, and 1 was Hispanic. Mean and median patient ages were 56 years and 57 years, respectively (range, 23 to 87 years).

Thirty-four tumors originated in the glomus jugulare and 6 in the glomus tympanicum. The precise site of origin within the temporal bone was unclear in an additional 2 lesions. Thirty-one patients had received no prior treatment at the time of presentation; 9 patients had lesions that were locally recurrent after surgery ( 6 patients), radiotherapy (1 patient), or surgery and radiotherapy ( 2 patients). Of the 8 patients who had local recurrences after prior surgery, 3 had undergone one operation and 5 had undergone two resections. All 3 patients with locally recurrent lesions after radiotherapy had been treated at other institutions. Patients were staged according to the classification of McCabe and Fletcher (Table 1) ${ }^{14,20}$ Eight patients (19\%) had stage I disease, $12(29 \%)$ had stage II disease, and $22(52 \%)$ had stage III tumors. Pretreatment evaluation included computed tomography (CT) in 25 patients and magnetic resonance imaging (MRI) in 11 patients. Pathologic confirmation of chemodectoma was obtained in 17 patients; the diagnosis was based on physical and radiographic findings in the remaining 23 patients.

Thirty-seven lesions were treated with radiotherapy alone, and 5 were managed with subtotal resection followed by postoperative radiotherapy for gross residual disease. The impression that gross residual disease remained after an operation was based on the observations of the surgeon, alone or combined with postoperative radiographic studies. All patients were irradiated with megavoltage equipment using either a continuous course (38 patients) or planned split-course ( 2 patients) technique. ${ }^{21}$ One patient discontinued radiotherapy, against medical advice, as a result of radiation mucositis after $21.8 \mathrm{~Gy}$ in 13 fractions. The dose ranged from $37.7 \mathrm{~Gy}$ to $50 \mathrm{~Gy}$ (mean, $42.9 \mathrm{~Gy}$ ) in the remaining 39 patients. All patients were treated with once-daily fractionation at 1.5 Gy to 1.96 Gy per fraction (mean, $1.77 \mathrm{~Gy}$ ). Twenty patients received $45 \mathrm{~Gy}$ in 25 fractions, 1.8 Gy per fraction, over 5 weeks. Patients were irradiated with cobalt 60 (22 patients), 6-MV x-rays (3 patients), 8-MV x-rays (1 patient), 20-MV x-rays (1 patient), or a combination of different beam energies (13 patients). Twenty patients were treated with an ipsilateral wedge-pair field arrangement, 17 patients with parallel opposed fields, 2 patients with a combination of the two, and 1 patient with parallel opposed fields and an ipsilateral boost field. In general, ipsilateral wedge-pair fields were used in well-lateralized lesions to minimize the dose to contralateral structures and reduce the likelihood of complications such as xerostomia. Patients with larger lesions that approached the midline were treated with parallel-opposed fields to reduce the risk of underdosing the medial extent of the tumor; the dose was weighted toward the involved side.

Patients had follow-up every 2 to 6 months for the first 5 years and annually thereafter. Follow-up scans were obtained as follows: CT (7 patients), MRI (7 patients), and both CT and MRI ( 7 patients). Patients treated during the early part of the study who remained clinically free of disease progression usually did not have CT or MRI as part of their follow-up.

Severe complications were defined as those that necessitated hospitalization or surgical intervention and/or were fatal. A moderately severe complication was defined as one that resulted in significant morbidity but was not severe enough to be classified as a severe complication. ${ }^{22}$

After irradiation, chemodectomas usually remain stable or partially regress. Therefore, for the purpose of this article, local control was defined as no evidence of disease progression and would include stable disease, partial regression, and complete regression. Thus, local control implies no evidence of disease progression based on the patient's symptoms, physical findings, and followup radiographic studies. Local control and complications were analyzed by the direct method. ${ }^{23}$ Exact test procedures were used to test differences between proportions. ${ }^{24}$ The likelihood of local control and cause-specific survival was also analyzed using the Kaplan-Meier product-limit method. ${ }^{25,26}$ All patients, including the one who discon-

Table 1. McCabe and Fletcher ${ }^{20}$ Classification of Temporal Bone Chemodectomas

I. Group I:

Tympanic tumor

II. Group II: Tympanomastoid tumor

III. Group III: Petrosal/extrapetrosal tumor
1. Absence of bone destruction

2. Intact eighth nerve

3. Intact jugular foramen

4. Absence of facial weakness

1. Bone destruction confined to mastoid

2. Facial nerve normal or paretic

3. Jugular foramen nerves intact

4. Superior bulb of the jugular vein involved by retrograde jugulography

1. Destruction on roentgenogram involving petrous bone, jugular fossa, and occipital bone

2. Positive retrograde jugulography

3. Jugular foramen syndrome

4. Presence of metastasis

5. Carotid arteriogram evidence of destruction of the petrous or occipital bones 
tinued irradiation prematurely, were included in the analyses of local control, cause-specific survival, and complications. Because prior irradiation compromises the ability to adequately reirradiate a chemodectoma, the subset of patients who had no prior radiotherapy was analyzed separately for the end points of local control and cause-specific survival.

\section{RESULTS}

\section{Local Control}

Local control versus stage and prior treatment is shown in Table 2. The 3 patients who developed a local recurrence did so at 2.3 years, 2.5 years, and 8.3 years, respectively, after treatment. Local control was obtained in $31(94 \%)$ of 33 previously untreated lesions, compared with $8(89 \%)$ of 9 previously treated chemodectomas $(P=$ .5247). All of the stage I and II tumors were controlled, as opposed to $19(86 \%)$ of 22 stage III lesions $(P=.1341){ }^{24}$ The likelihood of local control, calculated by the productlimit method, is depicted for all 42 lesions and for the subset of 39 tumors that had not been previously irradiated (Fig. 1). Four patients remain alive and continuously disease-free beyond 20 years (at 23 years, 23.9 years, 24.2 years, and 26.1 years, respectively). Local control versus irradiation dose and overall treatment time is shown in Figure 2. The patient who discontinued treatment at 21.8 Gy developed a local recurrence; otherwise, there is no obvious relationship between the likelihood of local control, radiation dose, and overall treatment time over the narrow range of doses and times used in this study. One patient underwent salvage surgery after disease progression and is currently disease-free 2.5 years after the operation.

\section{Survival}

The likelihood of cause-specific survival for the entire group of 40 patients and for the subset of 37 previously unirradiated patients is shown in Figure 3. Only 2 patients, one who had been previously irradiated and the one who discontinued treatment at $21.8 \mathrm{~Gy}$, died with progressing tumor. No patient in this series developed metastatic tumor in the neck nodes or in distant sites.

\section{Acute Complications}

One patient developed a severe radiation mucositis after $21.8 \mathrm{~Gy}$ in 13 fractions and became dehydrated, necessitating hospitalization and an unplanned interruption in the course of treatment. He subsequently refused to restart radiotherapy and did not complete treatment as scheduled. A second patient, who had received previous surgery and radiotherapy, developed delayed transient central nervous system syndrome, which resolved uneventfully. ${ }^{27}$

\section{Late Complications}

One patient developed a seventh nerve palsy 10 months after treatment, which resolved spontaneously. One patient who was treated with radiotherapy after two surgical procedures developed moderate trismus. One patient treated with subtotal resection and postoperative radiotherapy underwent removal of a cholesteatoma 13 months after treatment. One patient who underwent two prior operations as well as prior irradiation developed a partial optic neuropathy. The latter patient is the same one who developed delayed transient central nervous system syndrome. There were no fatal complications or radiationinduced malignancies. No other patient developed a cranial nerve deficit, after irradiation, in the absence of a local recurrence.

\section{DISCUSSION}

The efficacy of surgery and/or radiotherapy for temporal bone chemodectomas may be measured by local control of the tumor, survival, and complications. It is useful to consider lesions as either early-stage or advanced, particularly when evaluating the likelihood of complete resection and the risk of a treatment-related

Table 2. Local Control* versus Stage and Prior Treatment (no. controlled/no. treated) ${ }^{\dagger}$

\begin{tabular}{lccccc}
\hline & \multicolumn{5}{c}{ Prior Treatment } \\
\cline { 2 - 6 } McCabe-Fletcher & None & Surgery & Radiotherapy & $\begin{array}{c}\text { Surgery and } \\
\text { Radiotherapy }\end{array}$ & Total \\
\hline I & $7 / 7$ & $1 / 1$ & $0 / 0$ & $0 / 0$ & $8 / 8$ \\
II & $9 / 9$ & $3 / 3$ & $0 / 0$ & $0 / 0$ & $12 / 12$ \\
III & $15 / 17^{\ddagger}$ & $2 / 2$ & $1 / 1$ & $1 / 2$ & $19 / 22$ \\
Total & $31 / 33(94 \%)$ & $6 / 6$ & $1 / 1$ & $1 / 2$ & $39 / 42(93 \%)$ \\
\hline
\end{tabular}

*Calculated by the direct method.

tForty-two chemodectomas in $\mathbf{4 0}$ patients.

₹One patient (in whom local recurrence developed) discontinued treatment after $21.8 \mathrm{~Gy}$ in 13 fractions. 

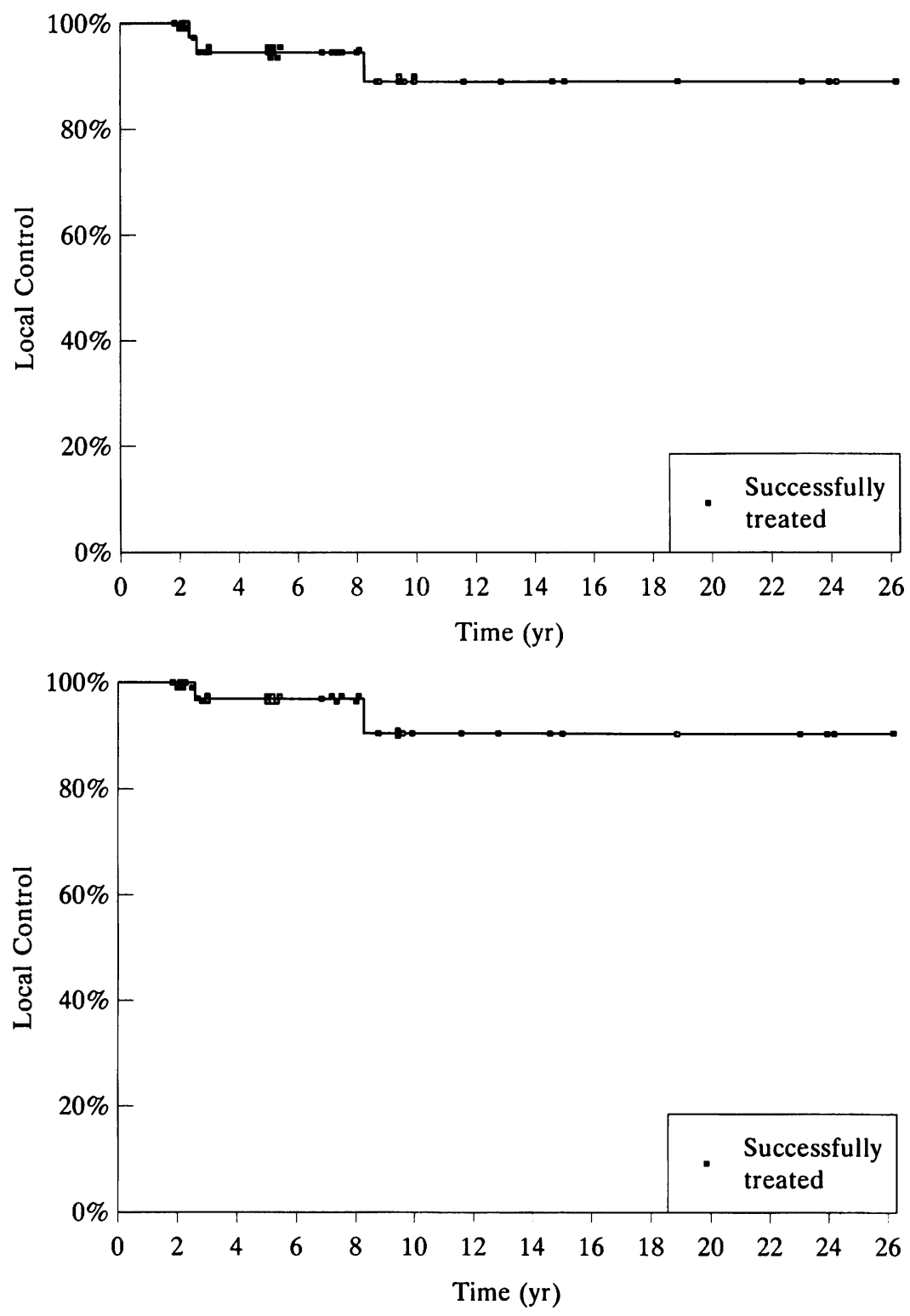

Figure 1. Local control calculated by the product-limit method. A: Overall group of 42 lesions in 40 patients. The probability of local control was $95 \%$ at 2.5 years, dropped to $89 \%$ at 8 years, and remained stable thereafter. B: Thirty-nine lesions in 37 patients who had not been previously irradiated. The likelihood of local control was $97 \%$ at 2.5 years, dropped to $91 \%$ at 8 years, and remained stable thereafter.

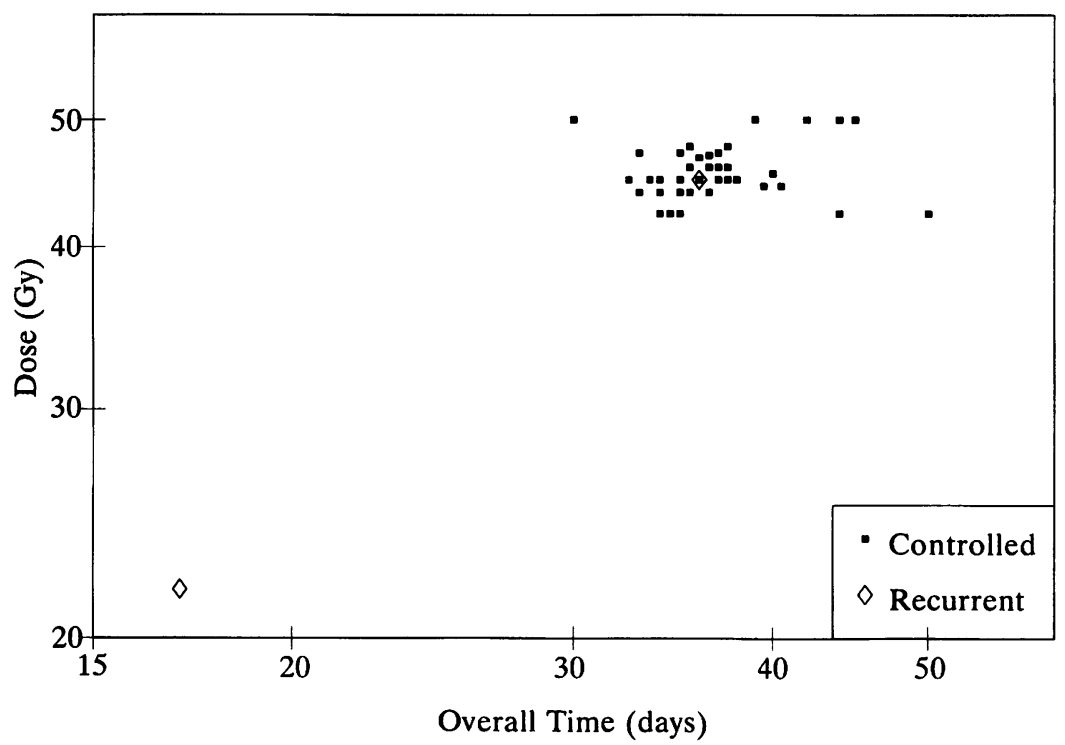

Figure 2. Local control versus radiotherapy dose (Gy) and overall treatment time (days) for the subset of 37 patients with 39 previously unirradiated chemodectomas. 
Figure 3. Cause-specific survival calculated by the product-limit method. A: Overall group of 40 patients. The probability of survival was $94 \%$ at 2.5 years and remained stable thereafter. B: Subset of 37 previously unirradiated patients. The likelihood of survival was $97 \%$ at 2.5 years and remained stable thereafter.
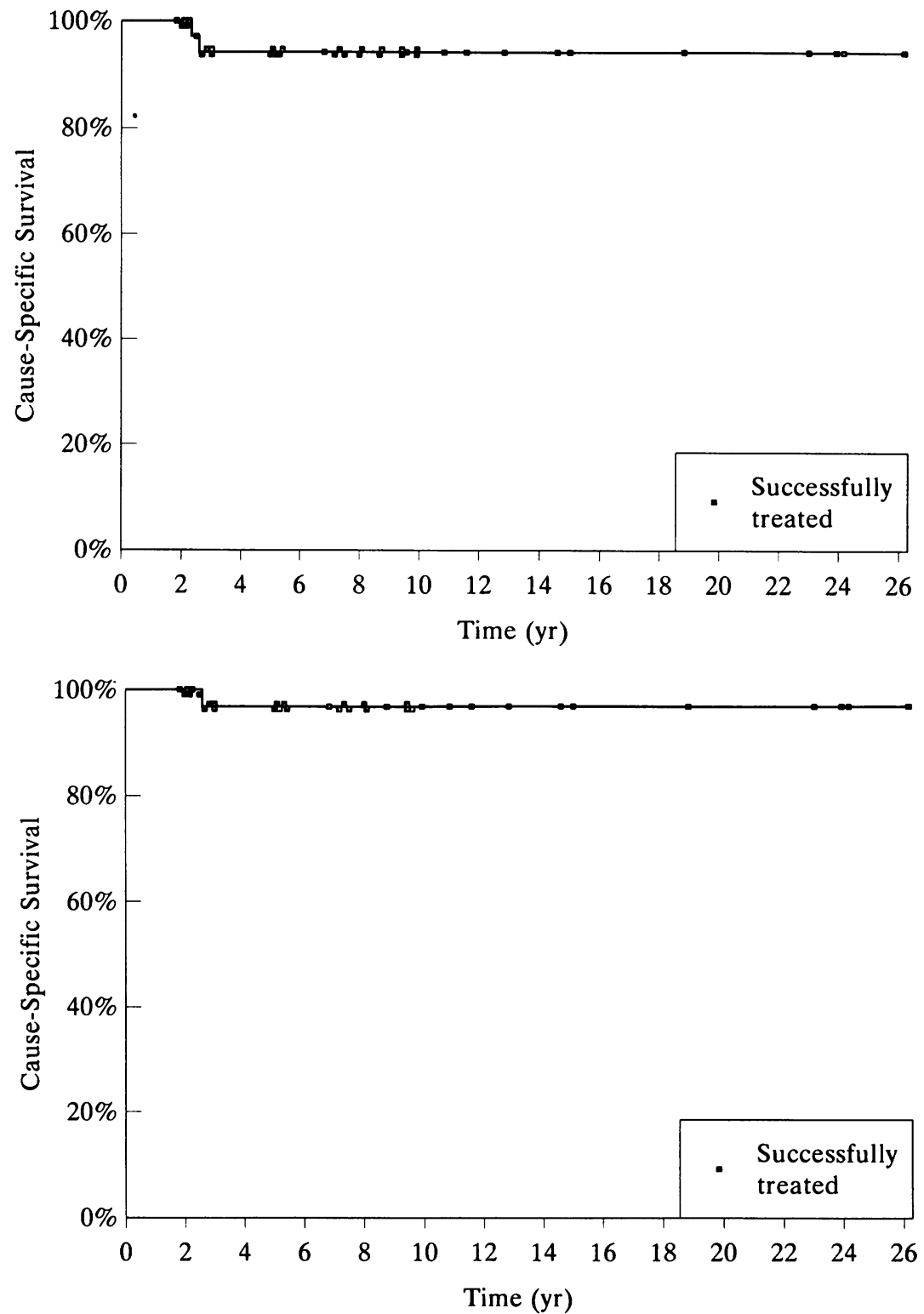

complication. Most authors employ the staging system outlined by McCabe and Fletcher ${ }^{20}$ or the classification proposed by Fisch. ${ }^{28}$ It is also necessary to stratify results according to whether the lesion has been previously untreated or is recurrent after prior surgery and/or irradiation. The reliability of various data depends on the length and adequacy of the follow-up. Other factors, such as cost and patient inconvenience, must also be considered.

\section{Local Control}

For the purposes of this discussion, local control after radiotherapy is defined as stable disease or tumor regression without evidence of regrowth. Although some have maintained that this is not the same as a "cure" after complete excision, it is essentially a cure from the patient's perspective ${ }^{19}$ Local control after an apparent com- plete resection implies that there is no evidence of tumor recurrence. Because chemodectomas may recur many years after treatment, it is preferable to analyze local control using the Kaplan-Meier product-limit method. ${ }^{23,25,26}$

Larner et al ${ }^{11}$ reported a series of 49 patients treated with surgery alone (20 patients), radiotherapy alone (15 patients), or surgery and postoperative radiotherapy (14 patients) at the University of Virginia between 1932 and 1985. Patients were staged according to the McCabe and Fletcher ${ }^{20}$ classification. Follow-up ranged from 5 to 31 years, with a median of 16.2 years. Surgery was used primarily for group 1 and 2 lesions, whereas radiotherapy tended to be used for group 3 lesions. Seventeen (85\%) of 20 patients undergoing surgery alone had an apparent complete resection. The rate of local control was $85 \%$ after surgery alone, $93 \%$ after radiotherapy alone, and $79 \%$ after surgery and postoperative radiotherapy. Powell et $\mathrm{al}^{12}$ reported a series of 64 patients with temporal bone 
chemodectomas treated at London's Royal Marsden Hospital between 1949 and 1985 with surgery alone (4 patients), surgery and radiotherapy (13 patients), radiotherapy alone (46 patients), or no treatment (1 patient). The actuarial rate of local control after radiotherapy alone was $90 \%$ at 10 years and $73 \%$ at 25 years. In all 13 patients treated with surgery and radiotherapy, the disease remained locally controlled from 1 to 22 years (median, 9 years); all 4 of the patients treated with surgery alone developed local recurrence. Wang et al ${ }^{5}$ reported a series of 32 patients with temporal bone chemodectomas treated with surgery (13 patients), radiotherapy (15 patients), or both (4 patients) at the University of Iowa between 1950 and 1981. Tumors were classified according to the system outlined by $\mathrm{McC}$ abe and Fletcher ${ }^{20}$; patients treated surgically tended to have earlier-stage lesions than those treated with radiotherapy alone. All 4 patients treated with surgery and radiotherapy had evidence of gross tumor at the time of irradiation. Follow-up ranged from 5 to 35 years. The local control rate after initial treatment was $46 \%$ after surgery alone and $84 \%$ after radiotherapy alone or combined with surgery. For those treated with surgery alone, local control was 5 of 8 for group I lesions and 1 of 5 for groups II and III. The ultimate local control rates, taking into account those successfully salvaged after local recurrence, were $85 \%$ for surgery alone and $84 \%$ after irradiation alone or combined with an operation.

Cummings et al ${ }^{17}$ reported 45 patients treated with irradiation for temporal bone chemodectomas at Toronto's Princess Margaret Hospital between 1958 and 1978. Treatment consisted of subtotal excision and radiotherapy (9 patients), radiotherapy alone for local recurrence after a prior operation (2 patients), or radiotherapy alone (34 patients). Follow-up ranged from 3 to approximately 25 years with a median follow-up of 10 years. Most patients received $35 \mathrm{~Gy}$ in 14 to 16 fractions over 3 weeks. Local control was obtained in 42 patients (93\%); all 3 patients who developed a local recurrence were successfully salvaged by an operation (1 patient) or a second course of radiotherapy ( 2 patients). Two of three local recurrences were thought to be secondary to a geographic miss. Konefal et $\mathrm{al}^{18}$ reported 23 patients treated with irradiation alone or combined with a subtotal resection at Washington University between 1961 and 1983. Patients received follow-up for up to 24 years after radiotherapy. Local control was observed in 19 patients (83\%); 2 of 4 patients who experienced a local recurrence received doses less than 41 Gy. Pryzant et al $^{14}$ reported 20 patients treated at Baylor University between 1965 and 1984. Patients were treated with radiotherapy alone (10 patients), surgery and radiotherapy (8 patients), or radiotherapy alone for a local recurrence after surgery ( 2 patients). Follow-up ranged from 3 to 23 years (mean, 11 years); 1 patient was lost to follow-up and was excluded from analysis of local control. Local control was achieved in $18(95 \%)$ of the remaining patients. In our series, the likelihood of local control at 20 years was $89 \%$ for the overall group of 42 lesions and $91 \%$ in the subset of 39 tumors that were previously unirradiated.

O'Leary et al ${ }^{1}$ reported 64 patients with glomus tympanicum tumors treated surgically at the House Ear Clinic in Los Angeles between 1957 and 1990. Minimum followup was approximately 6 months, and mean follow-up was 4.9 years. Three patients $(5 \%)$ had an incomplete resection, and the chemodectoma subsequently progressed in all 3. The rate of local control after surgery was $95 \%$. Woods et $\mathrm{al}^{29}$ reported 71 patients with glomus tympanicum tumors treated with surgery at The Otology Group in Nashville between 1970 and 1992. Although the length of follow-up was not stated, the minimum followup appeared to be less than 1 year and the maximum follow-up no more than 22 years. Sixty-five patients (92\%) underwent an apparent complete resection; subtotal resection was performed in 6 patients. Local recurrence developed in 2 additional patients at 3 and 4 years, respectively, so that 63 patients $(89 \%)$ had their tumors controlled by surgery.

Murphy and Brackmann ${ }^{4}$ reported 35 patients with glomus jugulare tumors who had surgery with an infratemporal fossa approach at the House Ear Clinic between 1981 and 1988. The length of follow-up was not stated but appeared to range from less than 1 year to a maximum of 8 years. Twenty-nine patients (83\%) underwent an apparent complete resection; 6 patients who had an incomplete resection had progressive tumor after the operation. Four of these 6 patients had received prior irradiation. Cece et $\mathrm{al}^{6}$ reported 17 patients with Fisch Group C and D glomus jugulare tumors who had surgery at New York's Mt. Sinai Medical Center from 1975 to 1985. The length of followup was not stated but appeared to range from approximately 1 to 12 years. The authors noted that the tumors in 16 patients $(94 \%)$ were locally controlled; the 1 patient who developed a local recurrence did so at 7 years. Jackson et $\mathrm{al}^{7}$ reported 11 patients with extensive glomus tumors of the temporal bone who had surgery with an infratemporal fossa approach at The Otology Group in Nashville between 1978 and 1981. The length of followup appeared to range from less than 1 year to a maximum of 4 years. Ten patients (91\%) underwent an apparent complete resection.

Woods et al ${ }^{29}$ reported, in an update of The Otology Group's experience, the management of 126 patients with glomus jugulare tumors (99 patients) and glomus vagale tumors (27 patients) who had surgery between 1971 and 1992. The authors excluded 26 patients originally treated for recurrence after prior treatment and 5 patients with "inadequate documentation," so that 95 patients who had surgery for previously untreated tumors were evaluable. The authors noted that patients with glomus vagale tumors were included in the series because these tumors "required standard skull base approaches for their removal." The Fisch stages for the 71 patients with glomus jugulare tumors were type B, 25 (35\%); type C, 20 (28\%); 
type $D_{1}, 22(31 \%)$; and type $D_{2}, 4(6 \%)$. Only $60(63 \%)$ of 95 patients were available for long-term follow-up, which ranged from 2 to 19 years (median, 5 years). An explanation for the lack of follow-up in the remaining 35 patients was not included. ${ }^{29,30}$ Forty-three patients had glomus jugulare tumors and 17 had glomus vagale tumors. Fortyeight tumors were completely removed and 4 were subtotally resected and irradiated postoperatively; all were locally controlled. The outcome of the remaining 8 patients was not stated.

Anand et $\mathrm{al}^{9}$ reported 20 patients with 29 chemodectomas with intracranial extension who had surgery at the University of Mississippi and Loyola University (Maywood, Illinois) between 1981 and 1990. Six of 20 patients were treated for a recurrence after prior surgery, which had been combined with irradiation and/or embolization in 5 of 6 cases. The mean follow-up was 4.5 years. Nineteen $(95 \%)$ of 20 patients underwent an apparent complete resection; 1 patient developed a local recurrence.

\section{Survival}

There is little information pertaining to the likelihood of absolute or cause-specific survival of patients treated for temporal bone chemodectomas. For such data to be meaningful, patients must have follow-up for extended periods of time and the analysis should be performed using the Kaplan-Meier product-limit method. ${ }^{25,26}$

Wang et $a^{5}$ reported 10 -year absolute survival rates of $78 \%$ after surgery alone and $76 \%$ after radiotherapy alone or combined with surgery. We noted that the probability of cause-specific survival at 20 years was $94 \%$ for the overall series of 40 patients.

\section{Complications}

Powell et al ${ }^{12}$ noted significant complications in 2 (4\%) of 46 patients treated with radiotherapy alone at the Royal Marsden Hospital; both patients developed a seventh cranial nerve palsy. Of note is that the patients received doses of $64 \mathrm{~Gy}$ and $66 \mathrm{~Gy}$, respectively, which are considerably higher than the current recommended doses for chemodectomas. There were no fatal complications or radiation-induced malignancies. Of 13 patients treated with combined surgery and irradiation, 1 patient died of pneumonia 3 months postoperatively, 1 patient died of chronic meningitis at 6 months, and 1 patient died of meningitis at 9 years. ${ }^{12}$ Konefal et a ${ }^{18}$ observed a significant complication in $1(4 \%)$ of 23 patients treated with irradiation alone or combined with surgery. The patient developed osteonecrosis of the temporal bone after $54 \mathrm{~Gy}$. Wang et $\mathrm{al}^{5}$ noted significant complications in $2(11 \%)$ of 19 patients treated with irradiation alone (15 patients) or combined with surgery (4 patients) at the University of
Iowa. One patient developed stenosis of the external auditory canal after $67 \mathrm{~Gy}$ in 28 fractions, which necessitated surgical intervention. A second patient died of radiation encephalopathy after $66.6 \mathrm{~Gy}$ in 37 fractions. Cummings et $\mathrm{al}^{17}$ noted that $2(4 \%)$ of 45 patients treated with radiotherapy developed severe complications: 1 patient underwent debridement for a bone necrosis after $58 \mathrm{~Gy}$ in 20 fractions, and another patient died of brain necrosis after $70 \mathrm{~Gy}$ in 16 fractions. The latter patient was accidentally given twice the intended dose. Additional complications included chronic otitis externa (4 patients), stenosis of the external auditory canal (1 patient), and chronic otitis media necessitating drainage ( 1 patient). Pryzant et al ${ }^{14}$ reported no significant complications in 20 patients treated with irradiation alone or combined with surgery at Baylor University.

O'Leary et $a^{1}{ }^{1}$ reported that $5(8 \%)$ of 64 patients who had surgery for glomus tympanicum tumors at the House Ear Clinic sustained complications: 3 patients had tympanic membrane perforations that required a tympanoplasty, 1 patient developed a cholesteatoma and underwent a revision mastoidectomy, and 1 patient developed seventh nerve weakness necessitating decompression. Woods et $\mathrm{al}^{29}$ reported that $7(10 \%)$ of 71 patients who had surgery for glomus tympanicum tumors at The Otology Group developed complications that included cholesteatoma ( 2 patients), tympanic membrane perforation ( 2 patients), seventh nerve paralysis (1 patient), wound infection (1 patient), and a stroke due to carotid artery injury (1 patient). Murphy and Brackmann ${ }^{4}$ reported the following incidence of new cranial nerve deficits in 35 patients undergoing resection of glomus jugulare tumors via an infratemporal fossa approach at the House Ear Clinic: ninth nerve (49\%), tenth nerve (37\%), and eleventh nerve $(51 \%)$. Woods et $\mathrm{al}^{29}$ reported an update of The Otology Group's series and included 95 patients with previously untreated glomus jugulare tumors (71 patients) and glomus vagale tumors (24 patients). Perioperative complications included cerebrospinal fluid leak (12\%), aspiration $(5 \%)$, aural infection (4\%), internal carotid artery blowout $(1 \%)$, meningitis $(2 \%)$, pulmonary embolus $(1 \%)$, acute respiratory distress syndrome $(1 \%)$, stroke $(2 \%)$, respiratory arrest $(1 \%)$, bacterial endocarditis $(1 \%)$, gastrointestinal bleeding (1\%), and death (2\%).

The rates of cranial nerve preservation in the 71 patients with glomus jugulare tumors were as follows: seventh nerve $(77 \%)$, eighth nerve $(49 \%)$, ninth nerve $(38 \%)$, tenth nerve $(52 \%)$, eleventh nerve $(52 \%)$, and twelfth nerve $(58 \%)$. Of note is that the incidences of preoperative cranial deficits were as follows: seventh nerve $(10 \%)$, eighth nerve $(21 \%)$, ninth nerve $(17 \%)$, tenth nerve $(23 \%)$, eleventh nerve $(13 \%)$, and twelfth nerve $(15 \%)$. The likelihood of cranial nerve preservation was inversely related to tumor stage.

Cece et $\mathrm{al}^{6}$ reported the incidence of complications in 17 patients who had surgery for Fisch group C or D glomus jugulare tumors at the Mt. Sinai Medical Center; 
all patients had new cranial nerve palsies or exacerbation of existing cranial nerve palsies. New cranial nerve palsies included the following: seventh nerve $(71 \%)$, ninth nerve (53\%), tenth nerve (41\%), and twelfth nerve (24\%). Thirteen patients $(76 \%)$ developed dysphagia and aspiration necessitating Teflon or Gelfoam injection (9 patients), cricopharyngeal myotomy (6 patients), and/or tracheostomy ( 2 patients). Additional complications included cerebrospinal fluid leak (2 patients), wound infection (1 patient), meningitis (1 patient), and chronic otorrhea due to a nonhealing mastoid cavity (1 patient). One patient (6\%) died several months after surgery because of aspiration. Anand et $\mathrm{al}^{9}$ noted that 10 of 20 patients who underwent surgery for chemodectomas with intracranial extension developed new cranial nerve deficits. Eight (40\%) of 20 patients required adjunctive procedures such as placement of a gold eyelid weight, cricopharyngeal myotomy, or vocal cord augmentation.

\section{CONCLUSIONS}

The likelihood of tumor control for chemodectomas of the temporal bone is high and is similar after either irradiation alone or surgery. There is no advantage to subtotal resection and radiotherapy compared with radiotherapy alone. Although some authors contend that patients treated with radiotherapy have not had sufficient follow-up to assure permanent disease control, follow-up in the radiotherapy series is as long as, or longer than, that reported in most surgical series. This is particularly true for patients with extensive temporal bone chemodectomas. In fact, follow-up data are not even provided in some of the previously cited papers from prominent institutions.

The decision to use irradiation or surgery is, therefore, based primarily on the risk of treatment complications. For early-stage temporal bone tumors with a low risk of surgical complications, we recommend primary resection for cure. For more advanced lesions, we recommend radiotherapy alone. Patients are treated with $45 \mathrm{~Gy}$ in 25 fractions over 5 weeks; there is no evidence that higher doses improve the already high likelihood of tumor control. The risk of significant complications after $45 \mathrm{~Gy}$ is negligible. Xerostomia secondary to irradiation of both parotid glands is minimized by the use of an ipsilateral wedged-pair field arrangement in most patients. ${ }^{15}$ The availability of three-dimensional treatment planning systems has improved our ability to use this treatment technique for larger tumors that might have required parallel opposed fields in the past. It should be remembered, however, that the most grievous error that can be made by the radiation oncologist in the treatment of this disease is inadvertent geographic miss; because the doses required for tumor control are modest and well tolerated by the normal tissues that are generally included within the treatment volume, "too-tight" treatment plan- ning is to be discouraged. The use of an inadequate irradiation treatment volume negates one of the major advantages of irradiation over surgery, namely, the ability to control tumor that is invading or in close proximity to vital structures without producing additional patient morbidity. While a dose of 45 Gy over 5 weeks is "safe," retreatment of a recurrence caused by a geographic miss with similar doses of radiation is attended by a significant risk of serious radiation injury.

\section{REFERENCES}

1. O'Leary MJ, Shelton C, Giddings NA, Kwarter J, Brackmann DE: Glomus tympanicum tumors: A clinical perspective. Laryngoscope 101:1038-1043, 1991

2. Jackson CG, Welling DB, Chironis P, Glasscock ME III, Woods CI: Glomus tympanicum tumors: Contemporary concepts in conservation surgery. Laryngoscope 99:875-884, 1989

3. Parsons JT, Mendenhall WM, Million RR, Stringer SP, Cassisi NJ Surgery vs radiation for chemodectomas: The debate continues. Oncology (Williston Park) 5(8):20, 1991

4. Murphy TP, Brackmann DE: Effects of preoperative embolization on glomus jugulare tumors. Laryngoscope 99:1244-1247, 1989

5. Wang M, Hussey DH, Doornbos JF, Vigliotti AP, Wen B: Chemodectoma of the temporal bone: A comparison of surgical and radiotherapeutic results. Int J Radiat Oncol Biol Phys 14:643648,1988

6. Cece JA, Lawson W, Biller HF, Eden AR, Parisier SC: Complications in the management of large glomus jugulare tumors. Laryngoscope 97:152-157, 1987

7. Jackson CG, Glasscock ME III, Harris PF: Glomus tumors: Diagnosis, classification, and management of large lesions. Arch Otolaryngol 108:401-406, 1982

8. Kim J, Elkon D, Lim M, Constable WC: Optimum dose of radiotherapy for chemodectomas of the middle ear. Int J Radiat Oncol Biol Phys 6:815-819, 1980

9. Anand VK, Leonetti JP, Al-Mefty O: Neurovascular considerations in surgery of glomus tumors with intracranial extensions. Laryngoscope 103:722-728, 1993

10. LaRouere MJ, Zappia JJ, Wilner HI, Graham MD, Lundy LB: Selective embolization of glomus jugulare tumors. Skull Base Surg 4:21-25, 1994

11. Larner JM, Hahn SS, Spaulding CA, Constable WC: Glomus jugulare tumors: Long-term control by radiation therapy. Cancer 69:1813-1817, 1992

12. Powell S, Peters N, Harmer C: Chemodectoma of the head and neck: Results of treatment in 84 patients. Int J Radiat Oncol Biol Phys 22:919-924, 1992

13. Springate SC, Haraf D, Weichselbaum RR: Temporal bone chemodectomas-comparing surgery and radiation therapy. Oncology (Williston Park) 5(4):131-137, 1991

14. Pryzant RM, Chou JL, Easley JD: Twenty year experience with radiation therapy for temporal bone chemodectomas. Int J Radiat Oncol Biol Phys 17:1303-1307, 1989

15. Dickens WJ, Million RR, Cassisi NJ, Singleton GT: Chemodectomas arising in temporal bone structures. Laryngoscope 92: 188-191, 1982

16. Friedland JL, Mendenhall WM, Parsons JT, Million RR, Cassisi $\mathrm{NJ}$ : Chemodectomas arising in temporal bone structures. Head Neck Surg 10:S52-S55, 1988

17. Cummings BJ, Beale FA, Garrett PG, et al: The treatment of glomus tumors in the temporal bone by megavoltage radiation. Cancer 53:2635-2640, 1984

18. Konefal JB, Pilepich MV, Spector GJ, Perez CA: Radiation therapy in the treatment of chemodectomas. Laryngoscope 97:13311335, 1987

19. Jackson CG: Neurotologic skull base surgery for glomus tumors: Section IX. Summary. Laryngoscope 103(suppl 60):71-72, 1993

20. McCabe BF, Fletcher M: Selection of therapy in glomus jugulare tumors. Arch Otolaryngol 89:156-159, 1969 
21. Parsons JT, Million RR, Bova FJ: A re-evaluation of split-course technique for squamous cell carcinoma of the head and neck. Int J Radiat Oncol Biol Phys 6:1645-1652, 1980

22. Taylor JMG, Mendenhall WM, Lavey RS: Dose, time, and fraction size issues for late effects in head and neck cancers. Int $J$ Radiat Oncol Biol Phys 22:3-11, 1991

23. Parsons JT, McCarty PJ, Rao PV, Mendenhall WM, Million RR: On the definition of local control. Int J Radiat Oncol Biol Phys 18:705-706, 1990

24. Ott L, Larson RF, Rexroat C, Mendenhall W: Statistics: A Tool for the Social Sciences. 5th ed. Boston: PWS-Kent, 1992

25. Kaplan EL, Meier P: Nonparametric estimation from incomplete observations. J Am Stat Assoc 53:457-481, 1958

26. SAS Institute Inc: SAS Technical Report P-179, Additional SAS/ STAT Procedures, Release 6.03. Cary, NC: SAS Institute Inc, 1988

27. Boldrey E, Sheline G: Delayed transitory clinical manifestations after radiation treatment of intracranial tumors. Acta Radiol Ther Phys Biol 5:5-10, 1966

28. Fisch U: Infratemporal fossa approach for glomus tumors of the temporal bone. Ann Otol Rhinol Laryngol 91:474-479, 1982

29. Woods CI, Strasnick B, Jackson CG: Neurotologic skull base surgery for glomus tumors: Section VIII. Surgery for glomus tumors: The Otology Group Experience. Laryngoscope 103 (suppl 60):65-70, 1993

30. Poe DS, Jackson CG, Glasscock ME, Johnson GD: Long-term results after lateral cranial base surgery. Laryngoscope 101:372378, 1991

The authors thank the research support staff of the Department of Radiation Oncology for their help with statistics, editing, and manuscript preparation.

\section{REVIEWER'S COMMENTS}

Chemodectomas of the temporal bone are rare tumors; nevertheless, the radiation oncologist will encounter this entity once in a while.

We must realize that radiation therapy has a definite role in the management of these histologically benign lesions; however, surgical excision remains the treatment of choice, particularly when tumors are confined to the site of origin. We must also realize that most patients referred for radiation therapy are those who are not good surgical candidates, or those with more advanced lesions, invading bony structures or causing cranial nerve deficits.

What remains controversial in the surgery and radiotherapy literature is the definition of cure and/or local control. Obviously, long-term follow-up is the key for the analysis of treatment outcome as well as morbidity, but the definition of local control in chemodectomas remains unknown.

Maria C. Jacobs, M.D. 